\title{
Recurrent Extubation Failure Following Neonatal Cardiac Surgery Is Associated with Increased Mortality
}

\author{
Shinya Miura ${ }^{1}$ (D) Warwick Butt ${ }^{1,2,3} \cdot$ Jenny Thompson ${ }^{1,2} \cdot$ Siva P. Namachivayam ${ }^{1,2,3}$ \\ Received: 29 November 2020 / Accepted: 31 March 2021 / Published online: 17 April 2021 \\ (c) The Author(s), under exclusive licence to Springer Science+Business Media, LLC, part of Springer Nature 2021
}

\begin{abstract}
Extubation failure (EF) following neonatal cardiac surgery is associated with increased mortality. Neonates who experienced EF twice or more (recurrent EF) may have worse outcomes than those who have a single EF or no-EF. The aims of this study are to investigate the in hospital mortality for neonates with recurrent EF compared to those with single or no-EF, and determine factors associated with recurrent EF. Neonates' $\leq 28$ days who underwent cardiac surgery from January 2008 to December 2019 were included. EF was defined as unplanned reintubation within $72 \mathrm{~h}$ after a planned extubation. 1187 (18 recurrent EF, 84 single EF and 1085 no-EF) neonates were included. Recurrent EF occurred in 18 (17.6\%) of 102 neonates undergoing a second extubation. The median time (IQR) to reintubation after the first and second extubations were similar, being 20.9 (3.3-45.2) versus $19.4(5.5-47) \mathrm{h}$. The reason for a second-time EF was respiratory in $39 \%$ and cardiovascular in 33\%. Recurrent EF and single EF was associated with increased mortality (odds ratio, 95\% confidence interval (CI) 23.5, 6.9-79.9) and (odds ratio, 95\% CI 5.2, 2.3-12.0) compared to no-EF. Based on the final model with risk adjustment, predicted mortality was $29.0 \%$ in recurrent EF, $6.5 \%$ in single EF, and $1.2 \%$ in no-EF. First-time EF due to cardiovascular compromise was associated with recurrent EF (odds ratio, 95\% CI 3.1, 1.0-9.7). This study confirmed that patients with recurrent EF have a high morality. Neonates with a cardiovascular reason for first-time EF are more likely to have a recurrent $\mathrm{EF}$ than those with other causes.
\end{abstract}

Keywords Extubation failure $\cdot$ Recurrent $\cdot$ Morality $\cdot$ Neonate $\cdot$ Cardiac surgery $\cdot$ Risk factor

\section{Background}

Previous studies have demonstrated that extubation failure (EF) occurs in $12-18 \%$ of neonates after cardiac surgery and is associated with increased mortality (8-30\%), prolonged length of stay in the pediatric intensive care unit (PICU) and hospital [1-4]. Several reports have shown that the etiology of EF was diverse including residual cardiac issues, respiratory or tracheal pathology, upper airway obstruction, diaphragmatic palsy, sepsis, or bleeding [4-6]. Some causes are amenable to simple treatments which will increase the

Shinya Miura

shin.nya1982@gmail.com

1 The Royal Children's Hospital Melbourne, Paediatric Intensive Care Unit, Melbourne, Australia

2 Murdoch Children's Research Institute, Melbourne, Australia

3 Department of Paediatrics, University of Melbourne, Melbourne, Australia likelihood of subsequent successful extubation, whilst other causes are more complex and may involve further surgery or be associated with complex congenital malformations [7].

We hypothesized that neonates experiencing EF twice or more (recurrent EF) may have a higher mortality than neonates experiencing EF once (single EF) or those experiencing no EF (non-EF). It was unclear as to the likely causes of each type of $\mathrm{EF}$ and whether this was important to ultimate outcome. Hence this study was done with the primary aim of investigating the hospital mortality among recurrent EF, single EF and non-EF. Secondary aims were to explore the reason of second-time $\mathrm{EF}$, and the association between first $\mathrm{EF}$ due to cardiovascular compromise and outcomes. 


\section{Methods}

\section{Study Design and Participants}

This is a single-center retrospective cohort study. The paediatric intensive care unit at the Royal Children's Hospital Melbourne is a 30-bed combined medical-surgical PICU. All patients after cardiac surgery are admitted to the cardiac pod in the PICU except premature neonates after patent ductus arteriosus ligation. We included all neonates who were 28 days or younger at cardiac operation and admitted to the PICU from January 1, 2008 to December 31, 2019. This study included only the first cardiac surgery for each neonate during the study period. Exclusion criteria included unplanned extubation, no extubation attempt, withdrawal prior to the extubation attempt and tracheostomy.

\section{Definitions and Data Collection}

EF was defined as unplanned reintubation within $72 \mathrm{~h}$ after planned extubation. The recurrent EF was defined as those who underwent EF twice or more after cardiac surgery. The single EF was defined as those who had EF once. Non-EF was defined as those who had no EF. The following data were retrospectively collected from the medical chart; patient characteristics and preoperative data (sex, gestation, weight, date of birth, date of surgery, date of PICU admission, cardiac diagnosis, comorbidities), operative data (risk adjustment in congenital heart surgery-1 (RACHS-1) category, cardiopulmonary bypass (CPB) time), peri-extubation data (the date and timing of extubation and reintubation, respiratory support post extubation), and outcomes (hospital death, PICU and hospital length of stay). RACHS-1 category was classified as low, medium, high RACHS-1 category based on the mortality in previous literatures. The cause of EF was chosen from a preset table of the etiology of EF by an investigator (S.M.) using information from the medical chart, laboratory result, radiology, echocardiography and other imaging, and conference report. If there are two or more etiologies, the primary one was decided in discussions among study investigators. In cases where there was any uncertainty as to the cause of $\mathrm{EF}$, the cause was ascertained by another investigator (S.P.N.) until two investigators reached an agreement. Among deceased patients without extubation attempt, they were considered to have treatment withdrawal if associated words such as palliation or treatment withdrawn due to fertility were documented in medical records.

\section{Statistical Analysis}

Collected data were presented as a number with the percentage for dichotomous variables and median with interquartile range (IQR) for continuous variables. For the analysis for hospital mortality among recurrent $\mathrm{EF}$, single EF and non$\mathrm{EF}$, a multivariable logistic regression model was developed by adjusting with preset study covariates (age, sex, gestation, chromosomal abnormality, CPB time, preoperative PICU admission, and RACHS-1 category). The predicted risk for hospital mortality by category of EF for the neonate after cardiac surgery was estimated from the final logistic model with covariates adjusted at the mean of observed values. Among 102 neonates who experienced EF once or more, the outcome was compared by the reason of reintubation (cardiovascular or the others) using a logistic regression model after adjusting with same study covariates. Two-tailed $\mathrm{p}$ values less than 0.05 were considered significant. STATA 14 (Stata Corp LLC, College Station, TX, USA) was used for all statistical analyses.

\section{Results}

During the study period, 1253 neonates underwent cardiac surgery. After excluding 56 neonates who met the exclusion criteria prior to the first extubation, 1197 underwent extubation. 9.4\% (112 out of 1197) neonates failed (Fig. 1). Ten of 112 reintubated neonates were excluded from the analysis prior to the second extubation attempt. 17.6\% (18 out of 102) experienced recurrent EF. 1187 neonates (18 recurrent EF, 84 single EF, 1085 non-EF) were included for the analysis. The characteristics of included patients are described in the Table 1. The overall hospital mortality was 3.3\%. Among the excluded 13 neonates who had an unplanned extubation, 11 were successful (non-EF), 1 failed and extubated successfully the next time and 1 died after 46 days in the PICU.

The crude mortality in recurrent EF, single EF, and nonEF were $33.3 \%$ (6 out of 18 ), $13.1 \%$ (11 out of 84 ), and $2.0 \%$ (22 out of 1085) (Fig. 2a). A logistic regression model showed higher hospital mortality in recurrent $\mathrm{EF}$ (odds ratio $23.5,95 \%$ confidence interval (CI) 6.9-79.9, $p<0.001)$ and single EF (odds ratio 5.2, 95\% CI 2.3-12.0, $p<0.001$ ) compared to non-EF after adjusting for study covariates. The predicted risk of hospital mortality by EF categories after adjustment of the study covariates are shown in the Fig. 2b, providing the predicted mortality of $29.0 \%$ in recurrent EF, $6.5 \%$ in single EF, and $1.2 \%$ in non-EF. Recurrent EF was associated with prolonged length of stay in PICU and hospital (Table 2). The Hosmer-Lemeshow goodness of fit test showed $\mathrm{p}$ value of 0.30 . When recurrent EF was compared to single EF among 102 neonate who experienced EF once or more, neonates with recurrent EF nearly had a fivefold increased odds of death (odds ratio 4.8 (95\% CI 1.2-19.2), $p=0.02$ ).

The proposed reason for recurrent EF was respiratory $(39 \%)$, cardiovascular $(33 \%)$, upper airway obstruction 
Fig. 1 Study flow. *After excluding 66 neonates who met exclusion criteria, 1187 neonates (18 with recurrent extubation failure, 84 with single extubation failure, and 1085 without extubation failure) were included for analysis. (Among the excluded 13 neonates who had an unplanned extubation, 11 were successful, 1 failed and extubated successfully the next time and 1 died after 46 days in the PICU)

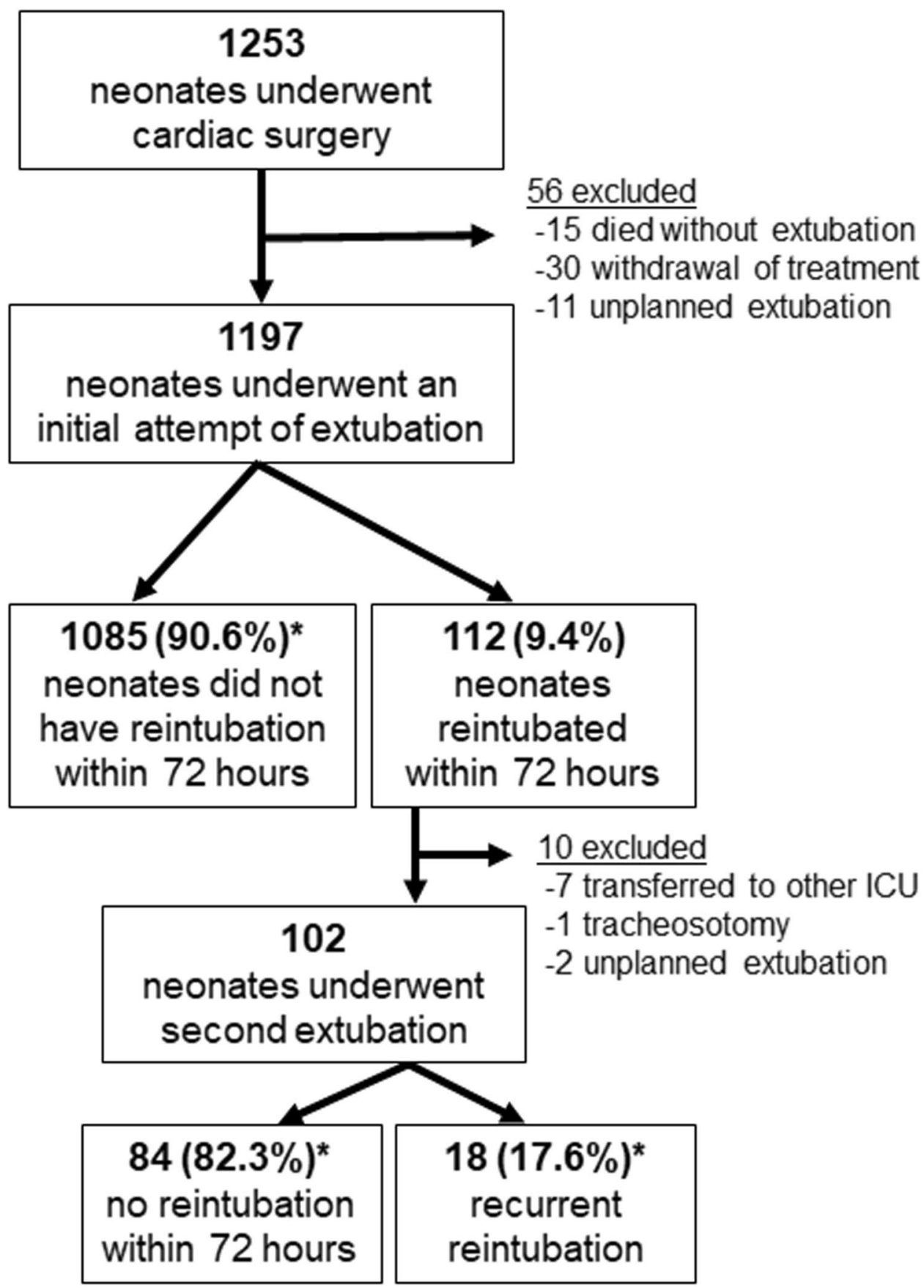

(6\%), and others (22\%) (Appendix Table 4). Characteristics of first-time and second-time EF is described in the Table 3. The median (IQR) time to reintubation after second extubation attempt was $19.4(5.5-47) \mathrm{h}$. Thirteen of eighteen neonates with recurrent EF were placed on continuous positive airway pressure (CPAP) immediately after second extubation while 119 of 1085 children with non-EF were extubated to CPAP. $39 \%$ (7 out of 18) of recurrent EF experienced second EF due to the same etiology as at first EF; ventricle dysfunction 2 , aortic valve regurgitation 1 , sepsis 2 , tracheomalacia 1 , tracheostenosis 1 .
Neonates who failed first extubation due to cardiovascular reasons had a higher odds of recurrent EF (odds ratio 3.1 (95\% CI 1.0-9.7), $p=0.048$ ) and higher hospital mortality (odds ratio 3.7 (95\% CI 1.1-12.4), $p=0.036$ ) compared to the other reasons for first EF after adjusting for study covariates.

The duration of mechanical ventilation in 102 neonates following first reintubation was 89 (68-134) h. The duration of mechanical ventilation from reintubation to second extubation attempt was associated with a hospital mortality of $13 \%$ in $<24 \mathrm{~h}, 6 \%$ in $24-48 \mathrm{~h}, 5 \%$ in $48-72 \mathrm{~h}, 7 \%$ in $72-96 \mathrm{~h}$, 
Table 1 Patient demographics

\begin{tabular}{|c|c|c|c|c|c|}
\hline Variable & All patients $n=1187$ & Recurrent $\mathrm{EF}^{\mathrm{a}} n=18$ & Single $\mathrm{EF}^{\mathrm{b}} n=84$ & Non-EF ${ }^{c} n=1085$ & $p$ \\
\hline Age at surgery, day & $7(4-12)$ & $4(3-8)$ & $5.5(3-10)$ & $7(4-12)$ & 0.01 \\
\hline Weight, kg & $3.3(2.9-3.6)$ & $3.1(2.6-3.7)$ & $3.3(3.0-3.7)$ & $3.3(2.9-3.6)$ & 0.50 \\
\hline Male & $731(62 \%)$ & $12(67 \%)$ & $54(64 \%)$ & $665(61 \%)$ & 0.78 \\
\hline Gestational age, week & $39(38-40)$ & $38(37-39)$ & $39(37-40)$ & $39(38-40)$ & 0.04 \\
\hline Chromosomal abnormality & $36(3 \%)$ & $1(6 \%)$ & $2(2 \%)$ & $33(3 \%)$ & 0.77 \\
\hline Preoperative ICU admission & $659(56 \%)$ & $14(78 \%)$ & $49(58 \%)$ & $596(55 \%)$ & 0.13 \\
\hline \multicolumn{6}{|l|}{ RACHS-1 category } \\
\hline $1-2$ & $187(16 \%)$ & $2(11 \%)$ & $8(10 \%)$ & $177(16 \%)$ & $<0.001$ \\
\hline $3-4$ & $833(70 \%)$ & $11(61 \%)$ & $52(62 \%)$ & $770(71 \%)$ & \\
\hline $5-6$ & $167(14 \%)$ & $5(28 \%)$ & $24(29 \%)$ & $138(13 \%)$ & \\
\hline Use of cardiopulmonary bypass & $998(85 \%)$ & $15(83 \%)$ & $76(90 \%)$ & $907(84 \%)$ & 0.25 \\
\hline Cardiopulmonary bypass time, min & $159(106-204)$ & $163(120-229)$ & $158.5(112.5-220.5)$ & $159(106-203)$ & 0.53 \\
\hline First extubation, POD & $3(2-5)$ & $7(3-8)$ & $4(2-6)$ & $3(2-5)$ & $<0.001$ \\
\hline Second extubation ${ }^{\mathrm{d}}$, POD & $8.5(6-13)$ & $10(8-14)$ & $8(6-12)$ & - & 0.06 \\
\hline
\end{tabular}

Values are provided as numbers (percentages) for categorical variables and as medians (interquartile ranges) for continuous variables. Categorical variables were analyzed using the chi-squared test and continuous variables were analyzed using the Kruskal-Wallis test to compare study characteristics between study groups

$I C U$ intensive care unit, RACHS risk adjustment for congenital heart surgery, $P O D$ postoperative day

${ }^{\mathrm{a}, \mathrm{b}, \mathrm{c}}$ Recurrent EF is neonates who experienced EF twice or more and single EF is neonates who experienced EF only once while non-EF is neonates experiencing no $\mathrm{EF}$

${ }^{\mathrm{d}}$ Data among 102 neonates with single or recurrent EF

and $36 \%$ in $>96 \mathrm{~h}$. Mechanical ventilation $>96 \mathrm{~h}$ was associated with increased mortality (odds ratio 14.9 (95\% CI $1.7-128.6 .7), p=0.01$ ). $30 \%$ (31 out of 102) had surgical interventions before second extubation attempt; open heart surgery $(n=3)$, regulation of the Blalock-Taussig shunt flow $(n=4)$, patent ductus arteriosus ligation $(n=1)$, hemostasis $(n=3)$, diaphragm plication $(n=4)$, aortopexy $(n=1)$, abdominal surgery $(n=3)$, chest washout $(n=7)$, exploration of heart $(n=2)$, and permanent pacemaker insertion $(n=3)$. The timing of surgical interventions following EF was on the same calendar day in $16,1-2$ days later in $5 ; 3-4$ days later in $5, \geq 5$ days in 5 .

\section{Discussion}

The main findings of this study are: (1) recurrent EF occurred in $18 \%$ of neonates who had a first EF, (2) hospital mortality incrementally increased as neonates experienced $\mathrm{EF}$, and (3) first EF due to cardiovascular reasons was associated with an increased risk of recurrent EF and mortality.

The association between recurrent EF and increased morality could be multi-factorial including patient-factors and ICU system-factors. Regarding patient-factors, we found that approximately $40 \%$ of neonates with recurrent EF failed their second extubation for the same reason, which was mainly structural or functional issues in the heart or airway.
Previous studies also reported these are common reasons of EF in neonates after cardiac surgery although only first the EF was reviewed [4]. In relation to ICU system-factors, neonates with congenital cardiac disease receiving multipleperiods of mechanical ventilation have an increased risk of nosocomial infection (sepsis, ventilator-associated pneumonia), lung injury secondary to prolonged positive pressure ventilation, deconditioning and weakness with respiratory muscle [8]. Frutos-Vivar et al. reported that among reintubated patients evolving infections after EF was associated with increased mortality [9]. Consequently, one neonate could be affected by multi-factors, especially considering the fact that mortality is often caused by multiple factors including both patient-factors and ICU system-factors.

Importantly, the cause of first-time EF can be a useful indicator to identify the high-risk cohort in neonates following cardiac surgery. In this study, cardiovascular reason for first EF was associated with recurrent $\mathrm{EF}$ and increased mortality. Thus, the ventilation plan tailored to neonates with the cause of EF is essential to avoid further ICU-related complications and recurrent $\mathrm{EF}$, which eventually increase the risk of morality. As an example, neonates experiencing EF with advanced heart failure may subsequently need further therapy including surgical interventions, long-term positive pressure support and nutrition plan for weeks or even months until the heart failure recovers. Some units are increasingly using the inotrope rotation therapy with 


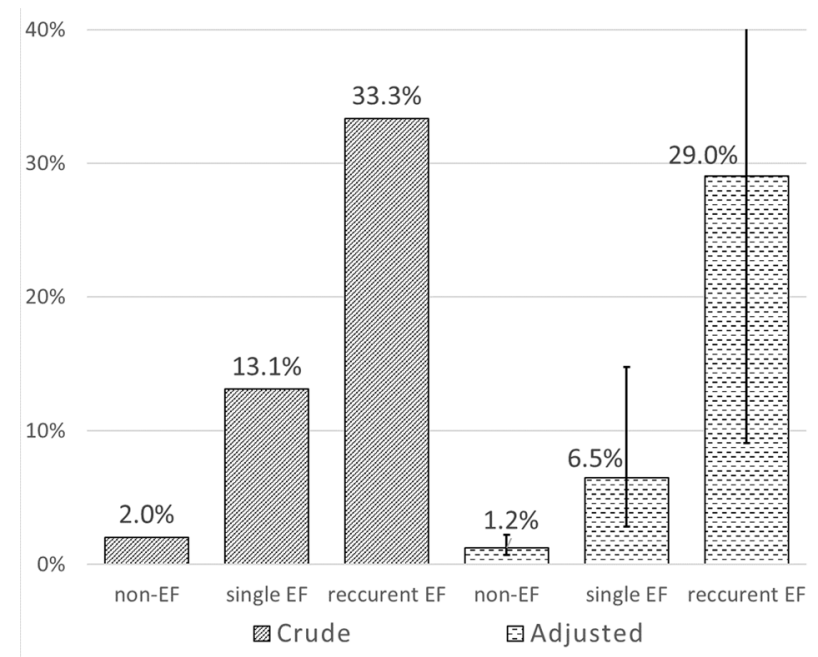

Fig. 2 Mortality by the number of extubation failure (no, once, twice or more) after neonatal cardiac surgery. $E F$ extubation failure. This figure shows crude (left) and predicted (right) risk of hospital mortality by the number of EF in neonates after cardiac surgery. 1173 neonates who had complete data were included in a logistic regression model to adjust the risk of hospital mortality for the study covariates (age, sex, gestation, chromosomal abnormality, cardiopulmonary bypass time, preoperative intensive care unit admission, RACHS-1 category). The predicted risk for hospital mortality by category of EF was estimated from the final logistic model with covariates adjusted at the mean of their observed values. Experiencing EF once was associated with mortality that was an absolute $5.3 \%$ higher than that if no EF: the probability of death in hospital was $6.5 \%$ (95\%CI 2.9-14.7\%) in single EF versus $1.2 \%$ (95\% CI $0.7-2.2 \%)$ in non-EF $(p<0.0001)$. Experiencing EF twice or more was associated with mortality that was approximately $22.5 \%$ higher than if experiencing EF only once: the probability of death in hospital was $29.0 \%$ (95\% CI 9.1-92.7\%) in recurrent EF versus 6.5\% (95\%CI 2.9-14.7\%) in single $\mathrm{EF}(p=0.03)$

long-term positive pressure support for heart failure [10]. The timing of surgical interventions following $\mathrm{EF}$ is also important point to consider since the timely interventions are associated with improved outcome compared to later operation if residual lesions are modifiable [11, 12]. By contrast, the timely ventilator weaning and extubation following stabilization of causes of EF may be the priority in neonates experiencing EF due to other causes (e.g., medically modifiable causes), especially considering the fact that prolonged mechanical ventilation after cardiac surgery is associated with a poor outcome and similarly in our study neonates who were ventilated $96 \mathrm{~h}$ or more after reintubation had a high mortality [1, 13]. For smoothing ventilation weaning, intensivists should be aware of latent issues deferring ventilator weaning, i.e., some respiratory and cardiovascular compromises could be difficult to recognize during ventilator support but would manifest at the weaning process, including diastolic dysfunction, hyperinflation associated with obstructive airway, reduced lung compliance compensated by baseline respiratory effort, and diaphragmatic dysfunction [8, 14-18]. There are a number of potentially useful tools to assess extubation-readiness during Spontaneous Breathing Trial (SBT) in high-risk patients including monitoring mixed venous oxygen saturation [19], B-type natriuretic peptide [20], respiratory workload [21], echocardiography [22], and ultrasound for diaphragm thickening and/or excursion [23].

Another important point to be studied is the setting of SBT in the second extubation. Thille et al. advocated that the risk of reintubation in high-risk population may become unacceptably high if they were assessed in the same way as unselected low-risk patients [16] For example, SBT with pressure support may have better ability to pick up patients ready for extubation than SBT with T-piece alone in unselected patients while SBT with pressure support may overestimate the extubation-readiness in high-risk patients, which randomized controlled trials could not have showed due to small cohort size of high-risk patients [16]. Among patients with difficult weaning, Cabello et al. demonstrated greater respiratory and cardiac workload in SBT with T-piece than SBT with pressure support [23]. Compared to commonlyused pressure support of $6-10 \mathrm{cmH} 2 \mathrm{O}$ during SBT at PICU $[24,25]$, Takeuchi et al. alarmed the possible overestimation of extubation-readiness by SBT with pressure support by demonstrating that SBT with the pressure support of 4 $\mathrm{cmH} 2 \mathrm{O}$ could replicate work of breathing post-extubation [21]. We need further evidence with regard to the setting
Table 2 The outcome by the number of extubation failure after neonatal cardiac surgery

\begin{tabular}{lcccc}
\hline & Recurrent $\mathrm{EF}^{\mathrm{a}} n=18$ & ${\text { Single } \mathrm{EF}^{\mathrm{b}} n=84}$ & $\mathrm{Non}^{-\mathrm{EF}^{\mathrm{c}} n=1085}$ & $p$ \\
\hline Hospital mortality, $n(\%)$ & $6(33 \%)$ & $11(13 \%)$ & $22(2 \%)$ & $<0.001$ \\
ICU stay, day & $26(18-66)$ & $10.5(7-21.5)$ & $4(3-7)$ & $<0.001$ \\
Hospital stay, day & $67(33-147)$ & $27.5(18-44.5)$ & $14(9-27)$ & $<0.001$ \\
\hline
\end{tabular}

Values are provided as numbers (percentages) for categorical variables and as medians (interquartile ranges) for continuous variables. Categorical variables were analyzed using the $\chi^{2}$ test and continuous

$E F$ extubation failure, $I C U$ intensive care unit

${ }^{a, b, c}$ Recurrent EF is neonates who experienced EF twice or more and single EF is neonates who experienced $\mathrm{EF}$ only once while non-EF is neonates experiencing no $\mathrm{EF}$

$p$ value was calculated by using the Kruskal-Wallis test to compare outcomes by category of EF 
Table 3 Characteristics of first and second extubation failure after neonatal cardiac surgery

\begin{tabular}{lcl}
\hline & First-time EF $n=102$ & Second-time EF $n=18$ \\
\hline $\begin{array}{l}\text { Extubation, postoperative day } \\
\text { Extubated to }\end{array}$ & $4(2-7)$ & $10(8-14)$ \\
CPAP & $47(46 \%)$ & $13(72 \%)$ \\
HHFNC & $18(18 \%)$ & 0 \\
Low-flow oxygen or nothing & $37(36 \%)$ & $5(28 \%)$ \\
Hours to reintubation $^{\text {Night-time extubation }}{ }^{\text {a }}$ & $20.9(3.3-45.2)$ & $19.4(5.5-47)$ \\
Night-time reintubation $^{\text {a }}$ & $26(25 \%)$ & $3(17 \%)$ \\
Etiology $^{\text {Upper airway obstruction }}$ & $59(58 \%)$ & $9(50 \%)$ \\
Respiratory & $11(11 \%)$ & $1(6 \%)$ \\
Cardiovascular & $44(43 \%)$ & $7(39 \%)$ \\
Others & $32(31 \%)$ & $6(33 \%)$ \\
\hline
\end{tabular}

Values are provided as numbers (percentages) for categorical variables and as medians (interquartile ranges) for continuous variables

$E F$ extubation failure, $C P A P$ continuous positive airway pressure, $H H F N C$ humidified high-flow nasal cannula

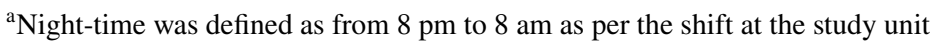

of SBT prior to extubation in re-intubated neonates after cardiac surgery.

There are several challenges for acquiring future evidence, as this cohort is characterized as a special population by following reasons. First, although tracheostomy is a common approach to smooth liberation from respiratory support and avoid complications by reducing sedation requirement and promoting rehabilitation among adults receiving prolonged ventilation, neonates after cardiac surgery may not as good candidates for tracheostomy because of the risk of surgical site infection and long-term airway complications. Second, the efficacy of post-extubation respiratory support could be different from other age groups; bi-level positive airway pressure is rarely feasible in this age as a nature of neonates breathing fast with small tidal volume; positive pressure of non-invasive respiratory support may not be delivered due to open mouth which is common in crying babies. Third, the etiology of EF and comorbidities varies from elder age group post cardiac surgery [26, 27]. Thus, these facts also highlight the importance of future studies in neonates suffering EF.

There are limitations in this study. First, it was a singlecenter study, limiting the generalizability of our findings to other centers as the mortality in recurrent EF can be influenced by patient-, surgery-, and intensive care-associated factors. Second, the setting of respiratory support pre and post extubation was not reviewed in this study, which could influence the outcome. Third, some potential cause of EF may be missed as some possible causes of EF (delirium, withdrawal, diaphragm muscle weakness, etc.) were difficult to detect in a neonatal study and may have been substituted by other causes like secretion or atelectasis. Forth, mortality is likely to be underestimated by excluding palliated neonates without extubation attempt. The decision of the palliation may vary depending on institutions. Lastly, as this study included twelve years of data, the chronological change in the use of non-invasive respiratory support (CPAP, and humidified high-flow nasal cannula therapy) may have influenced the study result.

\section{Conclusions}

Recurrent EF occurred in approximately $18 \%$ following first EF in neonatal cardiac surgery and it was associated with an even higher mortality than single EF. This study showed that recurrent $\mathrm{EF}$ is an important patient group in terms of high mortality. Neonates with a cardiovascular reason for first time EF are more likely to have a recurrent EF.

\section{Appendix}

See Tables 4 and 5. 
Table 4 Proposed reasons of extubation failure in second-time extubation attempt after neonatal cardiac surgery $(n=18)$

\begin{tabular}{lcll}
\hline Category & $\%$ & Reason & $n$ \\
\hline UAO & 6 & Secretion & 1 \\
Respiratory & 39 & Atelectasis & 2 \\
& & Pleural effusion & 1 \\
& & Malacia & 1 \\
& & Tracheobronchostenosis & 1 \\
& & Pneumonia & 1 \\
& & Other & 1 \\
Cardiovascular & 33 & Ventricular dysfunction & 2 \\
& & Pulmonary hypertension & 1 \\
& & Aortic valve regurgitation & 1 \\
Miscellaneous & 22 & Sepsis & 2 \\
& & Surgery & 2 \\
& & Necrotizing enterocolitis & 1 \\
& & Imaging & 1 \\
\hline
\end{tabular}

From the medical chart, laboratory result, radiology, echocardiography and other imaging, and conference report, the etiology among the category was chosen by a preset table of the etiology of extubation failure. If there are two or more etiologies, the primary one was decided in discussions among study investigators. Where there was doubt regarding ascertainment of the aetiology, a second expert's (S.P.N.) opinion was taken

$U A O$ upper airway obstruction
Table 5 Proposed reasons of extubation failure in first-time extubation attempt after neonatal cardiac surgery $(n=102)$

\begin{tabular}{|c|c|c|c|}
\hline Category & $\%$ & Reason & $n$ \\
\hline \multirow[t]{3}{*}{ UAO } & \multirow[t]{3}{*}{10} & Upper airway edema & 6 \\
\hline & & Vocal cord paralysis & 5 \\
\hline & & Unclear & 1 \\
\hline \multirow[t]{11}{*}{ Respiratory } & \multirow[t]{11}{*}{42} & Atelectasis & 7 \\
\hline & & Pleural effusion & 8 \\
\hline & & Pulmonary edema & 10 \\
\hline & & Diaphragm paralysis & 7 \\
\hline & & Malacia & 3 \\
\hline & & Pneumothorax & 2 \\
\hline & & Tracheo-bronchostenosis & 2 \\
\hline & & Pneumonia & 2 \\
\hline & & Muscle weakness & 2 \\
\hline & & Apnea & 2 \\
\hline & & Other & 4 \\
\hline \multirow[t]{7}{*}{ Cardiovascular } & \multirow[t]{7}{*}{31} & Ventricle dysfunction & 9 \\
\hline & & Pulmonary overcirculation & 8 \\
\hline & & AV valve regurgitation & 5 \\
\hline & & arrhythmia & 4 \\
\hline & & Shunt failure & 2 \\
\hline & & Aortic valve regurgitation & 1 \\
\hline & & Other & 7 \\
\hline Neuro & 2 & Over-sedation & 2 \\
\hline \multirow[t]{5}{*}{ Miscellaneous } & \multirow[t]{5}{*}{15} & Surgery & 7 \\
\hline & & Sepsis & 5 \\
\hline & & Bleeding & 3 \\
\hline & & Imaging & 1 \\
\hline & & Procedure & 1 \\
\hline
\end{tabular}

$A V$ valve atrioventricular valve

${ }^{\text {a }}$ Surgery; chest wound wash and debridement in five, pace maker insertion in two, direct left atrial line removal through sternotomy in one 
Acknowledgements We thank doctors Andrew Girgis and Peter Jardim for helping with data collection. Dr. S P Namachivayam is supported by a health professional research scholarship (award number 101003) from the National Heart Foundation of Australia.

Author Contributions All authors provided concept/idea/research design. SM provided data collection/data analysis/writing. JT provided and managed patient data. JT, SPN and WB revised and approved the manuscript.

\section{Declarations}

Conflict of interest The Authors declare that there is no conflict of interest.

Ethical approval This study was approved by the Royal Children's Hospital Melbourne Human Research Ethics Committee (reference no; 38300), and the need for informed consent was waived.

\section{References}

1. Benneyworth BD, Mastropietro CW, Graham EM et al (2017) Variation in extubation failure rates after neonatal congenital heart surgery across Pediatric Cardiac Critical Care Consortium hospitals. J Thorac Cardiovasc Surg 153:1519-1526. https://doi.org/ 10.1016/j.jtcvs.2016.12.042

2. Mastropietro CW, Cashen K, Grimaldi LM et al (2017) Extubation failure after neonatal cardiac surgery: a multicenter analysis. $\mathrm{J}$ Pediatr 182:190-196. https://doi.org/10.1016/j.jpeds.2016.12.028

3. Scodellaro T, McKenzie JM, D'Udekem Y et al (2017) Extubation failure is associated with increased mortality following first stage single ventricle reconstruction operation. Pediatr Crit Care Med 18:1136-1144. https://doi.org/10.1097/PCC.0000000000001334

4. Miura S, Hamamoto N, Osaki M et al (2017) Extubation failure in neonates after cardiac surgery: prevalence, etiology, and risk factors. Ann Thorac Surg 103:1293-1298. https://doi.org/10.1016/j. athoracsur.2016.08.001

5. Laudato N, Gupta P, Walters HL et al (2015) Risk factors for extubation failure following neonatal cardiac surgery. Pediatr Crit Care Med 16:859-867. https://doi.org/10.1097/PCC.0000000000000512

6. Gupta P, McDonald R, Gossett JM et al (2012) A single-center experience of extubation failure in infants undergoing the norwood operation. Ann Thorac Surg 94:1262-1268. https://doi.org/ 10.1016/j.athoracsur.2012.05.033

7. Epstein SK, Ciubotaru RL (1998) Independent effects of etiology of failure and time to reintubation on outcome for patients failing extubation. Am J Respir Crit Care Med 158:489-493

8. Thille AW, Cortés-Puch I, Esteban A (2013) Weaning from the ventilator and extubation in ICU. Curr Opin Crit Care 19:57-64. https://doi.org/10.1097/MCC.0b013e32835c5095

9. Frutos-Vivar F, Esteban A, Apezteguia C et al (2011) Outcome of reintubated patients after scheduled extubation. J Crit Care 26:502-509. https://doi.org/10.1016/j.jcrc.2010.12.015

10. Ryerson LM, Alexander PMA, Butt WW et al (2011) Rotating inotrope therapy in a pediatric population with decompensated heart failure. Pediatr Crit Care Med 12:57-60. https://doi.org/10. 1097/PCC.0b013e3181e2a437

11. Agarwal HS, Hardison DC, Saville BR et al (2014) Residual lesions in postoperative pediatric cardiac surgery patients receiving extracorporeal membrane oxygenation support. J Thorac Cardiovasc Surg 147:434-441. https://doi.org/10.1016/j.jtcvs.2013. 03.021
12. Nathan M, Gauvreau K, Liu H et al (2014) Outcomes differ in patients who undergo immediate intraoperative revision versus patients with delayed postoperative revision of residual lesions in congenital heart operations. J Thorac Cardiovasc Surg 148:2540 2547. https://doi.org/10.1016/j.jtcvs.2014.07.073

13. Peñuelas O, Frutos-Vivar F, Fernández C et al (2011) Characteristics and outcomes of ventilated patients according to time to liberation from mechanical ventilation. Am J Respir Crit Care Med 184:430-437. https://doi.org/10.1164/rccm.201011-1887OC

14. Brodsky JB (1988) Acute left ventricular dysfunction during unsuccessful weaning from mechanical ventilation. Anesthesiology 69:171-179

15. Buda AJ, Pinsky MR, Ingels NBJ et al (1979) Effect of intrathoracic pressure on left ventricular performance. N Engl J Med 301:453-459. https://doi.org/10.1056/NEJM197908303010901

16. Thille AW, Richard JCM, Brochard L (2013) The decision to extubate in the intensive care unit. Am J Respir Crit Care Med 187:1294-1302. https://doi.org/10.1164/rccm.201208-1523CI

17. Papanikolaou J, Makris D, Saranteas T et al (2011) New insights into weaning from mechanical ventilation: left ventricular diastolic dysfunction is a key player. Intensive Care Med 37:19761985. https://doi.org/10.1007/s00134-011-2368-0

18. Khemani RG, Sekayan T, Hotz J, Flink RC, Rafferty GF, Narayan Iyer CJLN (2017) Risk factors for pediatric extubation failure: the importance of respiratory muscle strength. Crit Care Med 45:e798-e805. https://doi.org/10.1097/CCM.0000000000002433

19. Jubran A, Mathru M, Dries D, Tobin MJ (1998) Continuous recordings of mixed venous oxygen saturation during weaning from mechanical ventilation and the ramifications thereof. Am J Respir Crit Care Med 158:1763-1769. https://doi.org/10.1164/ ajrccm.158.6.9804056

20. Mekontso-Dessap A, De Prost N, Girou E et al (2006) B-type natriuretic peptide and weaning from mechanical ventilation. Intensive Care Med 32:1529-1536. https://doi.org/10.1007/s00134-006-0339-7

21. Takeuchi M, Imanaka H, Miyano H, Kumon K (2000) Effect of patient-triggered ventilation on respiratory workload in infants after cardiac surgery. Anesthesiology 93:1238-1244. https://doi. org/10.1097/00000542-200011000-00017

22. Lamia B, Maizel J, Ochagavia A et al (2009) Echocardiographic diagnosis of pulmonary artery occlusion pressure elevation during weaning from mechanical ventilation. Crit Care Med 37:16961701. https://doi.org/10.1097/CCM.0b013e31819f13d0

23. Llamas-Álvarez AM, Tenza-Lozano EM, Latour-Pérez J (2017) Diaphragm and lung ultrasound to predict weaning outcome: systematic review and meta-analysis. Chest 152:1140-1150. https:// doi.org/10.1016/j.chest.2017.08.028

24. Newth CJL, Venkataraman S, Willson DF et al (2009) Weaning and extubation readiness in pediatric patients. Pediatr Crit Care Med 10:1-11. https://doi.org/10.1097/PCC.0b013e318193724d

25. Randolph AG, Wypij D, Venkataraman ST et al (2002) Effect of mechanical ventilator weaning protocols on respiratory outcomes in infants and children: a randomized controlled trial. J Am Med Assoc 288:2561-2568. https://doi.org/10.1001/jama.288.20.2561

26. Gupta P, Rettiganti M, Gossett JM et al (2016) Risk factors for mechanical ventilation and reintubation after pediatric heart surgery. J Thorac Cardiovasc Surg 151:451-458.e3. https://doi.org/ 10.1016/j.jtcvs.2015.09.080

27. Gaies M, Tabbutt S, Schwartz SM et al (2015) Clinical epidemiology of extubation failure in the pediatric cardiac icu: a report from the pediatric cardiac critical care consortium. Pediatr Crit Care Med 16:837-845. https://doi.org/10.1097/PCC.0000000000000498

Publisher's Note Springer Nature remains neutral with regard to jurisdictional claims in published maps and institutional affiliations. 